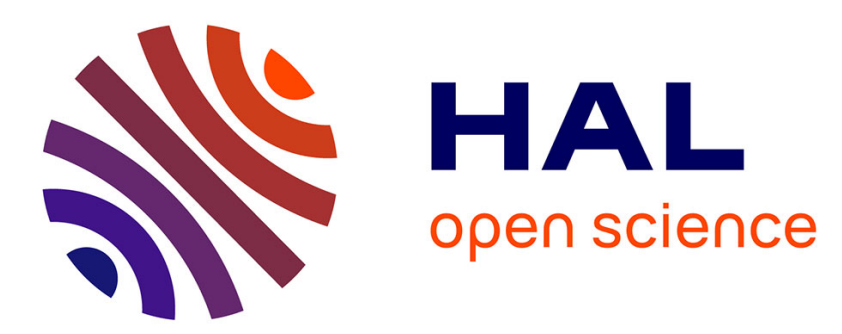

\title{
Electrohydrodynamic size stratification and flow separation of giant vesicles
}

Sigolène Lecuyer, William D. Ristenpart, Olivier O. Vincent, Howard A Stone

\section{To cite this version:}

Sigolène Lecuyer, William D. Ristenpart, Olivier O. Vincent, Howard A Stone. Electrohydrodynamic size stratification and flow separation of giant vesicles. Applied Physics Letters, 2008, 92 (10), pp.104105. 10.1063/1.2894182 . hal-02568934

\section{HAL Id: hal-02568934 \\ https://hal.science/hal-02568934}

Submitted on 10 May 2020

HAL is a multi-disciplinary open access archive for the deposit and dissemination of scientific research documents, whether they are published or not. The documents may come from teaching and research institutions in France or abroad, or from public or private research centers.
L'archive ouverte pluridisciplinaire HAL, est destinée au dépôt et à la diffusion de documents scientifiques de niveau recherche, publiés ou non, émanant des établissements d'enseignement et de recherche français ou étrangers, des laboratoires publics ou privés. 


\title{
Electrohydrodynamic size stratification and flow separation of giant vesicles
}

\author{
S. Lecuyer, W. D. Ristenpart, O. Vincent, and H. A. Stone ${ }^{\text {a) }}$ \\ School of Engineering and Applied Sciences, Harvard University, Cambridge, Massachusetts 02138, USA
}

(Received 22 October 2007; accepted 14 February 2008; published online 11 March 2008; publisher error corrected 19 March 2008)

\begin{abstract}
We demonstrate an electrohydrodynamic (EHD) technique for separating giant unilamellar vesicles by size in polydisperse suspensions. An oscillatory electric field $(\sim 30 \mathrm{~Hz})$ generates EHD flow around each vesicle close to an electrode. Nearby vesicles are entrained in the flow and the vesicles move toward one another. Upon aggregation, smaller vesicles are pulled underneath the larger vesicles, which ultimately lifts them off of the electrode. A brief spike in the electric field then serves to irreversibly adhere the bottom layer of smaller vesicles to the electrode, and the large vesicles are subsequently removed by flow. We demonstrate that a single application of this technique can remove more than $90 \%$ of the smallest vesicles (diameter $<20 \mu \mathrm{m}$ ) from a suspension of electroformed giant lipid vesicles. (C) 2008 American Institute of Physics.
\end{abstract}

[DOI: $10.1063 / 1.2894182$ ]

Unilamellar phospholipid vesicles are self-assembled, spherical molecular bilayers that separate a well-defined internal volume from the external environment. Giant unilamellar vesicles (GUVs), on the order of tens of micrometers in diameter, present an interesting analogy with living cells due both to their size and to the structural similarity between synthetic lipid bilayers and biological membranes. These characteristics have fueled interest in using GUVs as model systems in biophysics, ${ }^{1,2}$ as well as for novel applications including nanoreactors ${ }^{3}$ and designable drug carriers. ${ }^{4,5}$ Such applications often require precise control over the size of the vesicles. For example, with drug delivery and nanoreactors the quantity of active substance encapsulated is directly proportional to the vesicle volume. Likewise, the mean curvature of the vesicle membrane, which is crucial to many biological processes, ${ }^{6}$ is determined by the vesicle size.

Although there are established techniques for making nanometer-scale monodisperse vesicles (e.g., sonication, extrusion, ${ }^{8}$ and microfluidic generation $\left.{ }^{9}\right)$, the direct formation of monodisperse GUVs (e.g., using patterned electrodes ${ }^{10}$ or microfluidic devices ${ }^{11}$ ) has proven to be more challenging. The most widely used approach for synthesizing GUVs is electroformation, ${ }^{12}$ in which a low frequency electric field destabilizes planar hydrated lipid bilayers. This process yields polydisperse suspensions, with vesicles typically ranging in diameter from a few to several hundreds of micrometers. Unlike smaller unilamellar vesicles, GUVs are fragile, and common separation techniques (such as centrifugation or field flow fractionation), which work for smaller vesicles ${ }^{13}$ are not effective for GUVs. A method to separate GUVs from polydisperse suspensions is therefore desirable.

In this letter, we introduce a simple technique to separate GUVs by size, based on an electrohydrodynamic (EHD) aggregation phenomenon, followed by a transverse pressuredriven flow. EHD flows have been extensively studied in the context of rigid colloidal particles. ${ }^{14-18}$ An external electric field induces a dipole field around each colloid, which, in

\footnotetext{
a) Author to whom correspondence should be addressed. Electronic mail:
} has@seas.harvard.edu. turn, distorts the charge polarization layer near the electrode and gives rise to EHD flow (see Fig. 1). Since both the charge density near the electrode and the dipole field of the particle scale linearly with $E$, the resulting EHD flow scales as $E^{2}$. For colloidal particles with typical dipole strengths, the flow is directed radially inward along the electrode, 17,18 and the superposition of flows around adjacent particles causes them to move toward one another. Here, we establish that similar EHD flows are induced around unilamellar vesicles, but that they have strikingly different consequences for polydisperse suspensions: smaller vesicles are pulled preferentially underneath larger vesicles, ultimately lifting them off of the electrode entirely. We demonstrate that this effect can be exploited to selectively remove small vesicles from polydisperse suspensions, which dramatically increases the final fraction of larger GUVs. Since EHD separation does not require any additional equipment other than that already required for the electroformation of the vesicles, we believe this technique could be widely used to extract large GUVs preferentially from polydisperse suspensions.

Experiments were performed with dioleoylphosphatidylcholine (DOPC) (Avanti Lipids) vesicles, prepared using a standard electroformation procedure. ${ }^{12,19} \mathrm{~A}$ few microliters of DOPC dissolved in chloroform at $1 \mathrm{mg} / \mathrm{ml}$ were spread onto a clean indium tin oxide (ITO) slide and placed in a desiccation chamber overnight; the dry film was then hydrated with a $0.1 \mathrm{M}$ sucrose solution, and exposed for $4-5 \mathrm{~h}$ to a $1 \mathrm{~V}, 10 \mathrm{~Hz}$ ac electric field normal to the surface.

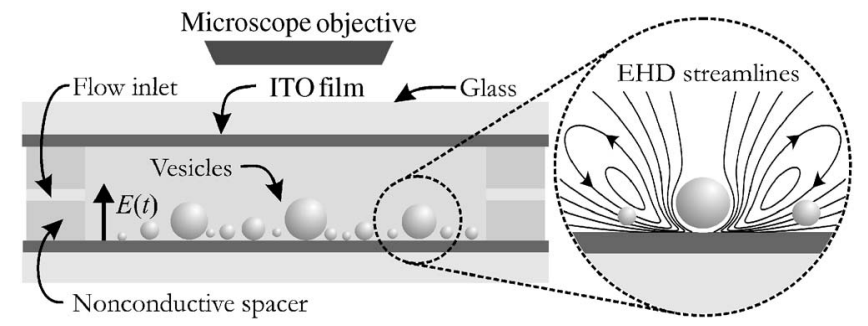

FIG. 1. Sketch of the experimental chamber (not to scale). Magnification: qualitative depiction of the EHD streamlines induced around an individual vesicle. 
The resulting polydisperse GUV suspension was then carefully pipetted out of the formation chamber and diluted in a $0.1 M$ glucose solution. This procedure yielded sucrose-filled GUVs suspended in glucose; a small volume of this solution was transferred into the chamber of the device sketched in Fig. 1. Two parallel glass slides coated with ITO $\left(R_{s}\right.$ $=4-8 \Omega$ ) served as electrodes and were separated by a nonconductive spacer (polydimethylsiloxane, $\sim 2 \mathrm{~mm}$ thick). The ITO was thoroughly cleaned then coated with a thin layer of bovine serum albumin to prevent vesicle adhesion. The index of refraction mismatch between sucrose and glucose facilitated phase-contrast microscopy, while the density mismatch caused the sedimentation of the GUVs toward the bottom electrode. Oscillatory electric fields were applied between the electrodes using a function generator and the resulting vesicle motion observed with a phase-contrast microscope (Leica DRM-IB).

For applied potentials below a threshold of approximately $1 \mathrm{~V}$, no discernible motion other than Brownian motion was observed. Above $1 \mathrm{~V}$, however, the resulting behavior depended sensitively on the applied frequency $f$. Upon application of fields with $f \geqslant 100 \mathrm{~Hz}$, the vesicles separated from one another in the direction transverse to the applied field. Occasionally, individual vesicles were observed to move on top of other vesicles such that they were aligned with the field. Both the transverse repulsion and vertical attraction are consistent with induced dipole-dipole interactions $;{ }^{20}$ similar behavior has been observed previously in suspensions of rigid colloids near electrodes. ${ }^{21,22}$

In contrast, for applied frequencies of $10 \mathrm{~Hz}<f$ $<100 \mathrm{~Hz}$, the vesicles moved toward one another, but the nature of the motion depended sensitively on the size disparity between the adjacent vesicles. Similarly sized vesicles tended to form planar clusters, in a manner comparable to the EHD aggregation of monodisperse rigid colloids reported previously. The overall motion is sketched qualitatively in Figs. 1(a) and 1(b) and demonstrated in movie 1 in the supplementary material. ${ }^{23}$ Small vesicles $(<10 \mu \mathrm{m})$ near larger vesicles $(>20 \mu \mathrm{m})$, however, exhibited two distinct behaviors: "orbiting" and "lifting." Orbiting was characterized by smaller vesicles recirculating in an axisymmetric toroidal flow centered around the larger vesicle; measurements of the orbiting speed indicated ${ }^{23}$ that the velocity scaled as $E^{2}$, consistent with EHD flow.

More often, however, groups of small vesicles exhibited a lifting behavior, wherein the smaller vesicles moved underneath the larger vesicles [Figs. 2(a)-2(c) and supplementary ${ }^{23}$ movie 2]. Some small vesicles immediately engaged in lifting, whereas others initially orbited for a few minutes before transitioning to lifting. The transition was sometimes quite abrupt, as a collection of multiple orbiting vesicles simultaneously rushed underneath the larger vesicle. More often the lifting was gradual, with small vesicles moving one by one underneath the big vesicle. As more vesicles progressed inward underneath the large vesicle, it was lifted upward until an entire layer of smaller vesicles lay between the large vesicle and the electrode [Fig. 2(c)]. The lifting process was reversible by either removing the field or increasing the frequency (supplementary ${ }^{23}$ movie 3 ).

Since the observations of orbiting (for $f<100 \mathrm{~Hz}$ ) and repulsion $(f>100 \mathrm{~Hz})$ suggest that both EHD flow and induced dipolar interactions affect the vesicle motion, presumably the lifting behavior is similarly driven by these forces.
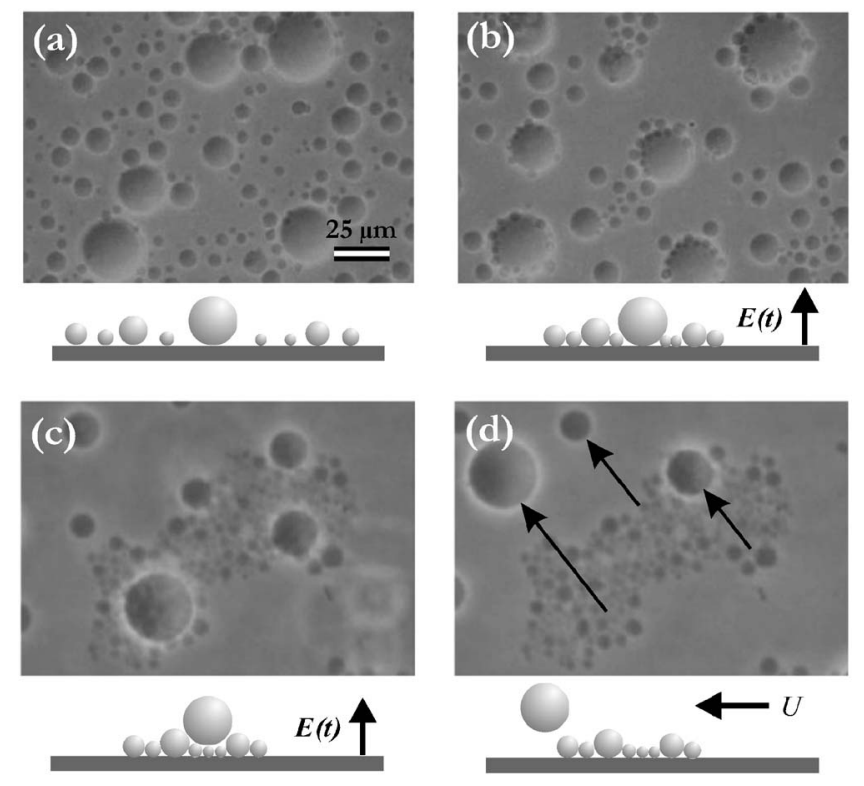

FIG. 2. Main stages of the EHD size separation process. (a) Prior to application of the field, the vesicles are arranged randomly near the electrode. (b) $30 \mathrm{~s}$ after application of a $10 \mathrm{~V}, 30 \mathrm{~Hz}$ ac field, smaller vesicles have aggregated around larger ones. (c) After $10 \mathrm{~min}$ of continuous application of the electric field, most of the larger vesicles have been lifted on top of clusters of smaller vesicles. (d) After a short dc pulse adheres the smaller vesicles to the electrode, a gentle pressure-driven flow (in direction of arrows) removes the larger vesicles.

The dipolar attraction is strongest directly underneath the large vesicle, and the EHD flow serves to sweep smaller vesicles along the electrode toward this region of strongest interaction. Because the force acting on each vesicle is affected by both the EHD flow and dipolar field induced around every other nearby vesicle, however, an accurate model of the entire lifting process will require detailed numerical calculations.

We focus here on demonstrating that lifting may be exploited to separate the vesicles by size, in the spirit of field flow fractionation processes. After the majority of the larger vesicles lifted off of the electrode, a dc field $(1 \mathrm{~V})$ was applied for $10 \mathrm{~s}$. This step caused the smaller vesicles to irreversibly adhere to the electrode, i.e., to become stuck in place. Because the larger vesicles were separated from the electrode by a layer of smaller vesicles, they did not stick and were free to move. Channels cut into the spacer on opposing sides of the chamber (cf. Fig. 1) provided a means for applying a pressure gradient through the device. In our apparatus (chamber volume of $\sim 20 \times 20 \times 2 \mathrm{~mm}^{3}$ ), a flow rate of $0.5 \mathrm{ml} / \mathrm{min}$ applied with an automatic syringe pump (Harvard apparatus) efficiently removed larger vesicles without stripping off the adhered small ones. The resulting flow pushed the large vesicles out into a collection chamber, while the irreversibly stuck smaller vesicles were left behind [Fig. 2(d) and supplementary ${ }^{23}$ movie 4]. Examples of vesicle suspensions before and after this separation process are shown in Fig. 3. The relative fraction of smaller vesicles is dramatically decreased by this technique; analysis of the size distribution before and after the separation process [Fig. 3(c)] shows that the fraction of vesicles of diameter below $20 \mu \mathrm{m}$ dropped from $93 \%$ to under $50 \%$. In terms of absolute numbers, more than $90 \%$ of the vesicles of diameter $20 \mu \mathrm{m}$ or under were removed. 

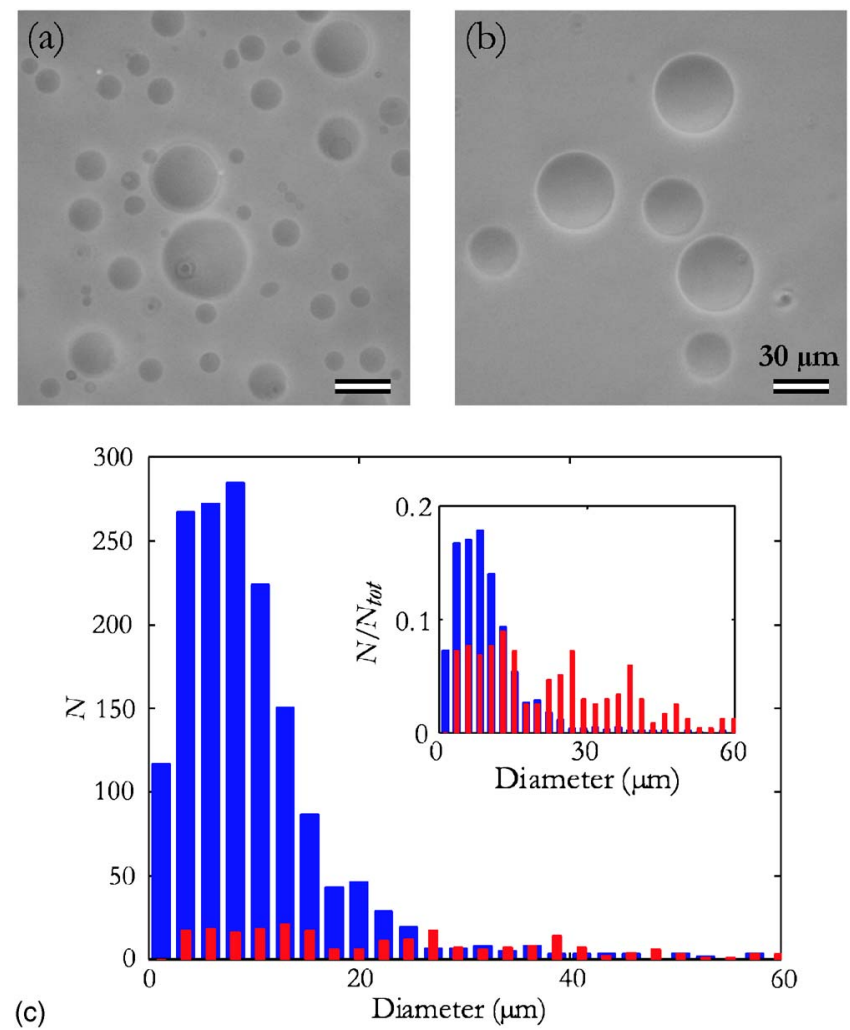

FIG. 3. (Color online) Representative images of the vesicles before (a) and after (b) the separation process. Scale bars are $30 \mu \mathrm{m}$. (c) Vesicle size distribution. Colors: blue $($ dark $)=$ before separation; red (light) $=$ after separation. Inset: same data normalized on the total number of vesicles. $N_{\text {tot }}$ $=1598$ and 234, respectively, before and after the separation (for the same number of images).

In summary, we demonstrated that EHD flows induce GUVs to undergo both aggregation and vertical size stratification near electrodes, and that this phenomenon can be exploited to preferentially separate the largest GUVs from polydisperse suspensions. A key advantage of this technique is its small space requirement, which will facilitate integration inside microfluidic lab-on-a-chip devices. Improvements to the separation efficiency might be achieved by repeating the EHD process sequentially or by optimizing the design of the cell. Although we have not observed any significant degradation of the ITO in our experiments, this is a frequent problem with low-frequency fields, which could motivate further optimization of the amplitude and duration of the electric fields applied. Improvements are so far primarily limited by lack of insight into the driving forces for the lifting. Despite extensive investigation, lifting has not been reported previously for polydisperse suspensions of rigid colloids, and it is unclear whether there is something unique about vesicles that promotes this behavior. Work is currently under way to address these questions.

S. Lecuyer and W. D. Ristenpart contributed equally to this work. The authors thank Harvard Nanoscale Science and Engineering Center for partial funding.

${ }^{1}$ J. Seddon and R. Templer, Structure and Dynamics of Membranes: From Cells to Vesicles (Elsevier, Amsterdam, 1995), Vol. 1A.

${ }^{2}$ U. Seifert, Adv. Phys. 46, 13 (1997).

${ }^{3}$ A. Graff, M. Winterhalter, and W. Meier, Langmuir 17, 919 (2001).

${ }^{4}$ T. Lian and R. J. Y. Ho, J. Pharm. Sci. 90, 667 (2001).

${ }^{5}$ X. Guo and F. C. Szoka, Acc. Chem. Res. 36, 335 (2003).

${ }^{6} \mathrm{R}$. Parthasarathy and J. T. Groves, Comput. Graph. 3, 24 (2007).

${ }^{7}$ M. Woodle and D. Papahadjopoulos, Methods Enzymol. 171, 193 (1989).

${ }^{8}$ M. Chow and M. Hope, Methods Enzymol. 367, 3 (2003).

${ }^{9}$ A. Jahn, W. Vreeland, D. DeVoe, L. Locascio, and M. Gaitan, Langmuir 23, 6289 (2007).

${ }^{10}$ P. Taylor, C. Xu, P. Fletcher, and V. Paunov, Chem. Commun. (Cambridge) 2003, 1732.

${ }^{11}$ H. Zhang, E. Tumarkin, R. Peerani, Z. Nie, R. M. A. Sullan, G. C. Walker, and E. Kumacheva, J. Am. Chem. Soc. 128, 12205 (2006).

${ }^{12}$ M. Angelova and D. Dimitrov, Faraday Discuss. Chem. Soc. 81, 303 (1986).

${ }^{13}$ B. A. Korgel, J. H. van Zanten, and H. G. Monbouquette, Biophys. J. 74, 3264 (1998).

${ }^{14}$ M. Trau, D. A. Saville, and I. A. Aksay, Science 272, 706 (1996).

${ }^{15}$ P. J. Sides, Langmuir 17, 5791 (2001).

${ }^{16}$ J. Kim, S. A. Guelcher, S. Garoff, and J. L. Anderson, Adv. Colloid Interface Sci. 96, 131 (2002).

${ }^{17}$ W. D. Ristenpart, I. A. Aksay, and D. A. Saville, Phys. Rev. E 69, 021405 (2004).

${ }^{18}$ W. D. Ristenpart, I. A. Aksay, and D. A. Saville, J. Fluid Mech. 575, 83 (2007).

${ }^{19}$ M. I. Angelova, S. Soléau, P. Méléard, J. Faucon, and P. Bothorel, Prog. Colloid Polym. Sci. 89, 127 (1992).

${ }^{20}$ A. P. Gast and C. F. Zukoski, Adv. Colloid Interface Sci. 30, 153 (1989).

${ }^{21}$ T. Gong and D. W. M. Marr, Langmuir 17, 2301 (2001).

${ }^{22}$ W. D. Ristenpart, I. A. Aksay, and D. A. Saville, Phys. Rev. Lett. 90, 128303 (2003).

${ }^{23}$ See EPAPS No. E-APPLAB-92-075810 for movies and additional data. This document can be reached through a direct link in the online article's HTML reference section or via the EPAPS homepage (http://www.aip.org/ pubservs/epaps.html). 